University of Nebraska - Lincoln

DigitalCommons@University of Nebraska - Lincoln

3-7-2008

\title{
Dependence of noise in magnetic tunnel junction sensors on annealing field and temperature
}

\author{
Sy-Hwang Liou \\ University of Nebraska-Lincoln, sliou@unl.edu \\ Rui Zhang \\ University of Nebraska - Lincoln \\ Stephen Russek \\ National Institute of Standards and Technology, Boulder, Colorado 80305, USA \\ L. Yuan \\ National Institute of Standards and Technology, Boulder, Colorado 80305, USA \\ Sean T. Halloran \\ National Institute of Standards and Technology, Boulder, Colorado 80305, USA \\ See next page for additional authors
}

Follow this and additional works at: https://digitalcommons.unl.edu/physicsliou

Part of the Physics Commons

Liou, Sy-Hwang; Zhang, Rui; Russek, Stephen; Yuan, L.; Halloran, Sean T.; and Pappas, David P., "Dependence of noise in magnetic tunnel junction sensors on annealing field and temperature" (2008). SiHwang Liou Publications. 97.

https://digitalcommons.unl.edu/physicsliou/97

This Article is brought to you for free and open access by the Research Papers in Physics and Astronomy at DigitalCommons@University of Nebraska - Lincoln. It has been accepted for inclusion in Si-Hwang Liou Publications by an authorized administrator of DigitalCommons@University of Nebraska - Lincoln. 
Authors

Sy-Hwang Liou, Rui Zhang, Stephen Russek, L. Yuan, Sean T. Halloran, and David P. Pappas

This article is available at DigitalCommons@University of Nebraska - Lincoln: https://digitalcommons.unl.edu/ physicsliou/97 


\title{
Dependence of noise in magnetic tunnel junction sensors on annealing field and temperature
}

\author{
S. H. Liou ${ }^{\text {a) }}$ and Rui Zhang \\ Department of Physics and Astronomy and Nebraska Center for Materials and Nanoscience, \\ University of Nebraska-Lincoln, Lincoln, Nebraska 68588, USA
}

Stephen E. Russek, L. Yuan, Sean T. Halloran, and David P. Pappas

National Institute of Standards and Technology, Boulder, Colorado 80305, USA

(Presented on 7 November 2007; received 5 October 2007; accepted 16 November 2007; published online 7 March 2008)

The minimum detectable field of magnetoresistive sensors is limited by their intrinsic noise. Magnetization fluctuations are one of the crucial noise sources and are related to the magnetization alignment at the antiferromagnetic-ferromagnetic interface. In this study, we investigated the low frequency noise of magnetic tunnel junctions (MTJs) annealed in the temperature range from 265 to $305^{\circ} \mathrm{C}$ and magnetic fields up to $7 \mathrm{~T}$, either in helium or hydrogen environments. Our results indicate that the magnetic fluctuators in these MTJs changed their frequency based on annealing field and temperature. The noise of the MTJs at low frequency can be reduced by annealing in high magnetic field $(7 \mathrm{~T})$ and further improved by annealing in a hydrogen environment. (C) 2008 American Institute of Physics. [DOI: 10.1063/1.2837659]

\section{INTRODUCTION}

Sensor noise is a crucial parameter in low-field applications. Magnetic and nonmagnetic noises sources contribute to the noise of a magnetoresistive based devices. ${ }^{1-10}$ These two noise processes can be identified by comparing the noise level and sensitivity at various applied magnetic fields. At low frequencies, these noise sources are either frequency independent or frequency dependent. Frequency independent noises include thermal resistance noise, shot noise, and thermomagnetic fluctuation noise. Thermal resistance noise, also known as Johnson or Nyquist noise $V_{J N}$, results from a thermal smearing of the density of states near the Fermi level. It is given by $V_{J N}=\sqrt{4 k_{B} T R}$, where $R$ is the array resistance, $T$ is the junction temperature, and $k_{B}$ is Boltzmann's constant. Shot noise $\left(V_{S N}\right)$ is due to the stochastic nature of electron transport with an applied current. It can be expressed by $V_{\mathrm{sn}}=\sqrt{2} V_{s} q R / N$, where $V_{s}$ is the voltage applied to the junction, $N$ is number of series junctions in each leg of the array, and $q$ is the electron charge. Intrinsic magnetic noise is generated by thermal magnetization fluctuations $\left(V_{M N}\right)$. It can be written as

$$
V_{\mathrm{mn}}=\frac{\Delta R V_{s}}{R H_{k}} \sqrt{\frac{k_{B} T \alpha \mu_{0}}{2 \pi N \Omega M_{s} \gamma}},
$$

where $\Delta R$ is the total array resistance change in the measurement range, $H_{k}$ is the anisotropy field of the free layer, $\alpha$ is the damping parameter, $\mu_{0}$ is the permittivity of vacuum, $\Omega$ is the volume of the magnetic tunnel junction (MTJ) free layer, $M_{s}$ is the saturation magnetization of the free layer, and $\gamma$ is the gyromagnetic ratio. ${ }^{7}$ Another kind of noise, $1 / f$ noise, also called flicker noise or excess noise, is frequency dependent. It arises from the coupling of electronic processes with magnetization fluctuations and can be characterized by Hooge's formula, ${ }^{11,12}$

\footnotetext{
${ }^{a)}$ Electronic mail: sliou@unl.edu
}

$$
S_{f}=\frac{\alpha_{H} V_{s}^{2}}{N A f^{\beta}},
$$

where $\alpha_{H}$ is the material-specific Hooge parameter, $A$ is the junction area, and $\beta$ is the exponent of the $1 / f$ noise spectrum.

At high frequencies Johnson and shot noise are dominant where the $1 / f$ noise dominates at low frequencies. The magnetic noise is very sensitive to the dynamics and disorder in the magnetic system. Magnetization fluctuations are related to the magnetization alignment at the antiferromagnetic (AFM)-ferromagnetic (FM) interface. In this study, we investigated the low frequency noise of MTJs annealed in the temperature range from 265 to $305^{\circ} \mathrm{C}$ and magnetic fields up to $7 \mathrm{~T}$ either in helium or hydrogen environments. Annealing aims at improving the degree of the magnetization alignment at the AFM-FM interface as well as the quality of FM and AFM layers. This improvement depends on annealing temperature, magnetic field, and environment. It is known that the noise spectrum is very sensitive to these parameters. ${ }^{1}$

\section{EXPERIMENT}

All the MTJs used in our study have the following structure: $5 \mathrm{~nm} \mathrm{Ta} / 5 \mathrm{~nm} \mathrm{Cu} / 10 \mathrm{~nm} \mathrm{Ir}_{20} \mathrm{Mn}_{80} / 2 \mathrm{~nm} \mathrm{Co}_{90} \mathrm{Fe}_{10} /$ $0.85 \mathrm{~nm} \quad \mathrm{Ru} / 3 \mathrm{~nm} \quad \mathrm{Co}_{60} \mathrm{Fe}_{20} \mathrm{~B}_{20} / 1.4 \mathrm{~nm} \quad \mathrm{Al}_{2} \mathrm{O}_{3} / 2 \mathrm{~nm}$ $\mathrm{Co}_{90} \mathrm{Fe}_{10} / 28 \mathrm{~nm} \mathrm{Ni}{ }_{80} \mathrm{Fe}_{20} / 5 \mathrm{~nm} \mathrm{Ta} / 5 \mathrm{~nm} \mathrm{Ru}$. The junctions were patterned into ellipses with a size of either 5 $\times 7.5 \mu \mathrm{m}^{2}$ (area of $59 \mu \mathrm{m}^{2}$ and eccentricity of 0.74 ) or 10 $\times 15 \mu \mathrm{m}^{2}$ (area of $236 \mu \mathrm{m}^{2}$ and eccentricity of 0.74 ). They were configured into 64 element symmetric bridges. Figure 1 shows a sample with each junction having a size of 5 $\times 7.5 \mu \mathrm{m}^{2}$. The magnetization of the reference layer (the $\mathrm{Co}_{60} \mathrm{Fe}_{20} \mathrm{~B}_{20}$ layer in the synthetic antiferromagnet) is pinned along the sensor short axis. The noise measurement system was in a shielded environment to avoid picking up unwanted 


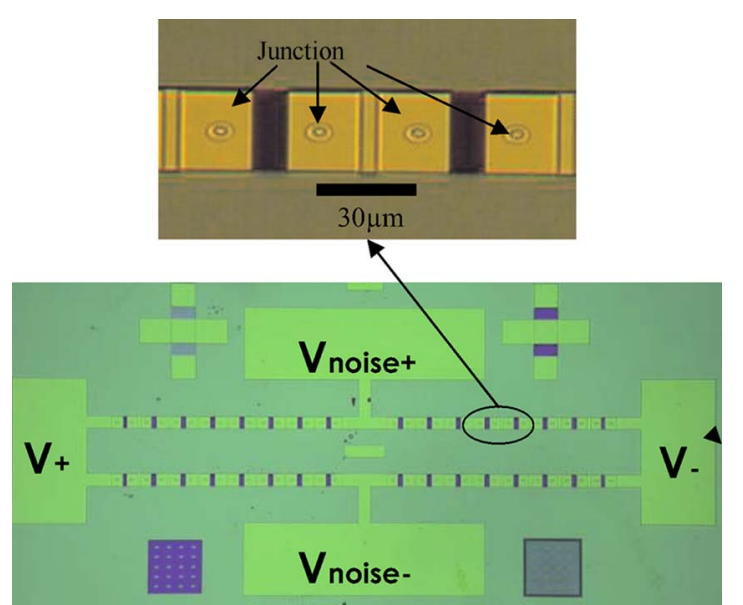

FIG. 1. (Color online) The picture of the MTJ bridge with junction size of $5 \times 7.5 \mu \mathrm{m}^{2}$. Inset is enlarged picture of those MTJs.

external magnetic field fluctuations. The sensor voltage bias was supplied by a battery to minimize noise. A low noise preamplifier with a gain of 100 was used. The noise spectra of the MTJ are taken at room temperature with a constant bridge voltage bias of $1.0 \mathrm{~V}$ supplied by a battery, which provided a bias of approximately $31 \mathrm{mV} / \mathrm{junction}$. The noise from the preamplifier was about

$$
V_{\mathrm{amp}}=\sqrt{\frac{4 \times 10^{-16} V_{s}^{2}}{f}+\left(4.8 \times 10^{-9} V_{s}\right)^{2}} .
$$

There was also a parallel capacitance due to both the MTJ devices and the amplification circuit. This causes a capacitive roll-off of $A_{R C}=1 /\left[1+(2 \pi f R C)^{2}\right]$, where $C$ is the capacitance of the circuit (on the order of $3 \times 10^{-11} \mathrm{~F}$ ). The details of the measurement system are described in reference. ${ }^{3}$ The total noise of the sensor can be expressed as $V_{t n}=\sqrt{A_{R C}^{2}\left(S_{\mathrm{mn}}+S_{\mathrm{Jsn}}+S_{f}\right)+V_{\mathrm{amp}}^{2}}$, where the $S_{\mathrm{mn}}$ is the intrinsic magnetic noise power spectrum, $S_{\text {Jsn }}$ is the Johnson and shot noise power spectrum, and $S_{f}$ is the $1 / f$ noise power spectrum. We have used the above equation to fit our experimental data.

\section{RESULTS AND DISCUSSIONS}

First, we investigated the low frequency noise of an MTJ bridge with $5 \times 7.5 \mu \mathrm{m}^{2}$ ellipses annealed at $265^{\circ} \mathrm{C}$ with different magnetic fields. As shown in Fig. 2(a), the magnetoresistance versus magnetic field curve shows that the MTJ annealed at $265^{\circ} \mathrm{C}$ in a $7 \mathrm{~T}$ magnetic field is slightly less hysteretic than that of the one annealed at $265^{\circ} \mathrm{C}$ in a $0.5 \mathrm{~T}$ magnetic field. As shown in Fig. 2(b), the sensors, after having been annealed at $7 \mathrm{~T}$ and $265{ }^{\circ} \mathrm{C}$ for $15 \mathrm{~min}$ and measured at zero bias field, showed an improvement in the noise spectrum by about 2.5 times in the range of 1 to $10 \mathrm{~Hz}$. There were only small changes at a higher frequency range (the small bump at about $20 \mathrm{~Hz}$ that deviates from the $1 / f$ noise spectrum). These changes are related to the change of magnetic fluctuators in the MTJ due to annealing. The source of noise in MTJs can be magnetic or nonmagnetic in origin, which can be distinguished by measuring in different magnetic fields. There is about an order of magnitude reduction of the low frequency noise spectrum when the MTJ mea-
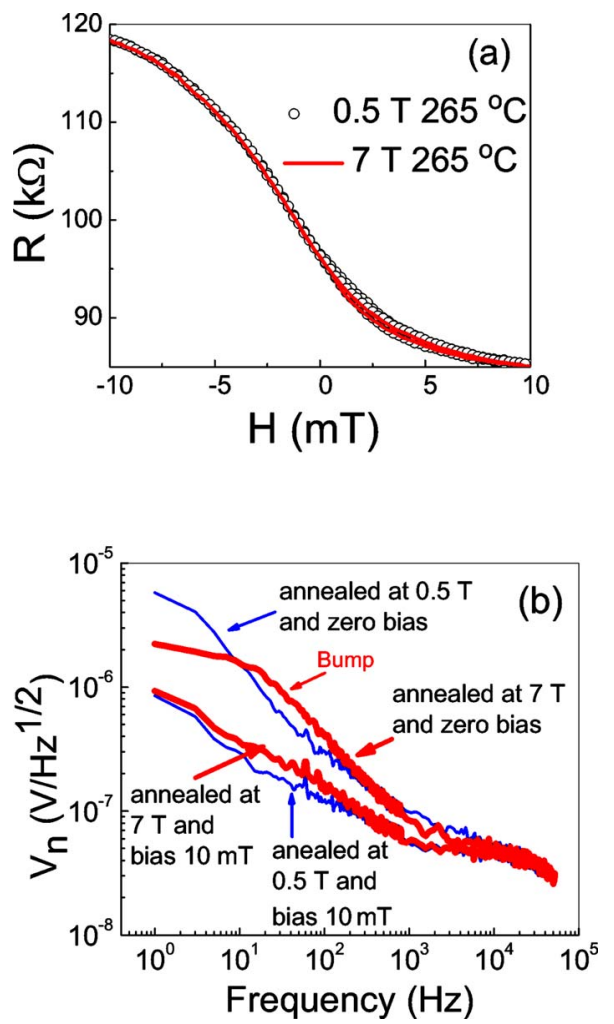

FIG. 2. (Color online) (a) The $R$ vs $H$ curve of the MTJ bridge with junction size of $5 \times 7.5 \mu \mathrm{m}^{2}$ annealed at $265{ }^{\circ} \mathrm{C}$ in fields of 7 and $0.5 \mathrm{~T}$. (b) The low frequency noise spectrum of MTJs with junction size of $5 \times 7.5 \mu \mathrm{m}^{2}$ annealed in 0.5 and $7 \mathrm{~T}$ fields in a helium environment.

sured with a $10 \mathrm{mT}$ bias magnetic field. (In this magnetic field the magnetization of the free layer is parallel to that of the pinned layer.)

As shown in Fig. 3, we observed a similar low frequency noise spectrum for a MTJ array with $10 \times 15 \mu \mathrm{m}^{2}$ ellipses annealed at $265^{\circ} \mathrm{C}$ with different magnetic fields. That is, the noise spectrum was reduced by about two times in the range of $1-10 \mathrm{~Hz}$ after annealing at $7 \mathrm{~T}$ and $265^{\circ} \mathrm{C}$ for $15 \mathrm{~min}$ and measured at zero bias field. There is also a shift of magnetic fluctuators to a higher frequency (the small bump at about $200 \mathrm{~Hz}$ that deviates from the $1 / f$ noise spectrum) due to annealing at $7 \mathrm{~T}$ and $265^{\circ} \mathrm{C}$. There were no significant changes of the magnetoresistance versus magnetic field curve when the same MTJ array was annealed at $285^{\circ} \mathrm{C}$ in a $0.5 \mathrm{~T}$ magnetic field. As shown in Fig. 4, there

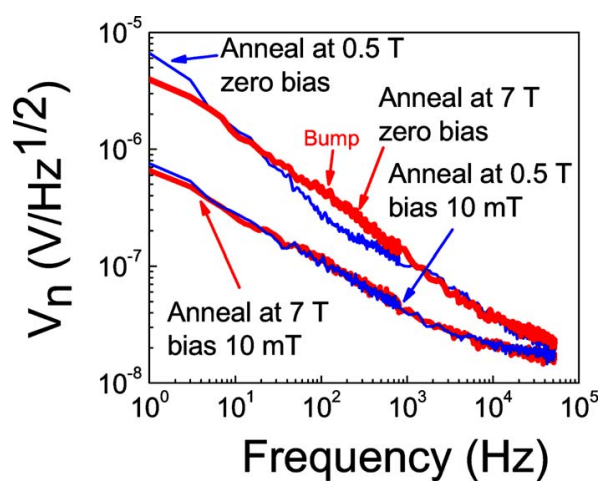

FIG. 3. (Color online) The noise spectrum of MTJ bridge with junction size of $10 \times 15 \mu \mathrm{m}^{2}$ annealed at $265^{\circ} \mathrm{C}$ in fields of 0.5 and $7 \mathrm{~T}$ in a helium environment. 


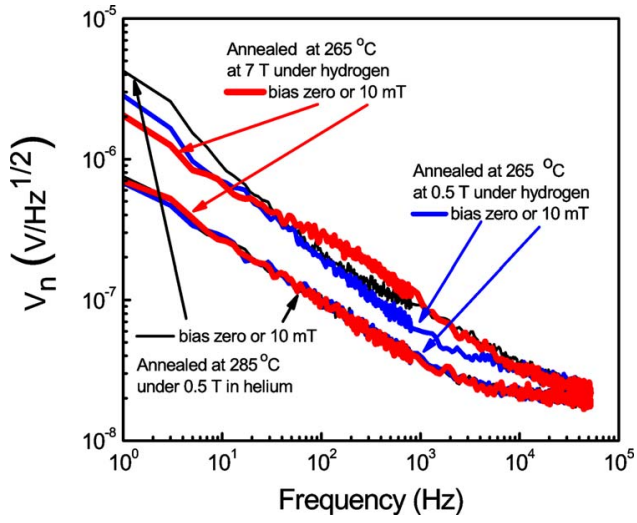

FIG. 4. (Color online) The noise spectrum of MTJ bridge with junction size of $10 \times 15 \mu \mathrm{m}^{2}$ annealed at $265{ }^{\circ} \mathrm{C}$ in fields of 0.5 and $7 \mathrm{~T}$ in a hydrogen environment. (The test sample was annealed at $265^{\circ} \mathrm{C}$ in fields of 0.5 and $7 \mathrm{~T}$ and then annealed at $285^{\circ} \mathrm{C}$ in a field of $0.5 \mathrm{~T}$ in a helium environment before the hydrogen treatment.)

were also no significant changes in the noise spectrum of the MTJ annealed at $7 \mathrm{~T}$ and $265^{\circ} \mathrm{C}$ (as shown in Fig. 3) and then annealed at $285^{\circ} \mathrm{C}$ in a $0.5 \mathrm{~T}$ magnetic field. For MTJ samples annealed at $305^{\circ} \mathrm{C}$, the magnetoresistance ratio decreased and the low frequency noise increased.

To reduce the noise level further, we investigated the annealing of MTJ samples in a hydrogen atmosphere. In this study, we used the above MTJs (size of $10 \times 15 \mu \mathrm{m}^{2}$ ) that were already annealed at $285{ }^{\circ} \mathrm{C}$ and in $0.5 \mathrm{~T}$ and then annealed them again in a different magnetic field in hydrogen gas at $265^{\circ} \mathrm{C}$. As shown in Fig. 4, the noise level of the MTJ annealed in hydrogen gas and $0.5 \mathrm{~T}$ was reduced to half of that at low frequency $(1 \mathrm{~Hz})$ compared with the original sample (which was annealed at $285^{\circ} \mathrm{C}$ in $0.5 \mathrm{~T}$ ) and the noise level of the MTJ annealed in hydrogen gas at $7 \mathrm{~T}$ was reduced to one third that at low frequency $(1 \mathrm{~Hz})$ compared with the original sample. From the facts that there is only very small changes of the MR loop before and after hydrogen annealing of MTJ samples in high magnetic field, it indicates that there is no significantly altering of the magnetization alignment of the AFM and FM layers at the interface. Hydrogen annealing of MTJ samples in high magnetic field may only remove some of defects and change the pinning sites in the free layer as well as the pinning layer which leads to the shift of magnetic fluctuators to higher frequencies and the reducing of the noise at low frequency. By optimizing the annealing temperature in $\mathrm{H}_{2}$ we may further improve the noise floor at low frequency in the future.

Figure 5 shows the fitting of the noise spectra, which provides values for the Hooge parameter $\alpha_{H}$ for the MTJ bridge annealed in different conditions. In these fits, we obtained $\alpha_{H}$ 's of $3.5 \times 10^{-8}, 1.5 \times 10^{-8}$ and $7.8 \times 10^{-9} \mu \mathrm{m}^{2}$ for MTJs after they were annealed at $285^{\circ} \mathrm{C}$ in $0.5 \mathrm{~T}$ in helium, $265^{\circ} \mathrm{C}$ in hydrogen in $0.5 \mathrm{~T}$, and $265^{\circ} \mathrm{C}$ in hydrogen in $7 \mathrm{~T}$, respectively. This clearly indicates a reduction of $1 / f$ noise by annealing the MTJ in a hydrogen environment and high magnetic field. In addition to the $1 / f$ component, there is a bump (or bumps) shown in the noise spectrum. These bumps indicate that there are some strong discrete magnetic fluctuators in the MTJ, shown as Lorentzian-like peaks in the

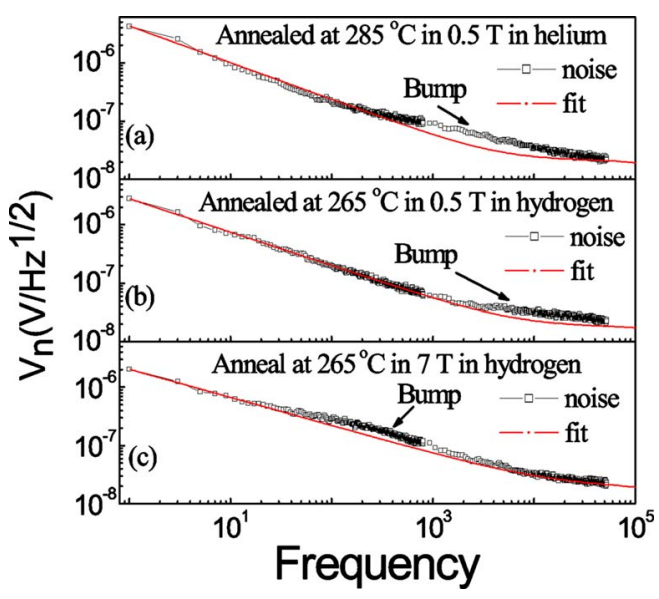

FIG. 5. (Color online) Model fits and noise spectra of the MTJ bridge with junction size of $10 \times 15 \mu \mathrm{m}^{2}$ annealed in a hydrogen environment (measured in zero bias magnetic field).

noise spectra. The bump got smaller after the MTJ bridge was annealed at $265{ }^{\circ} \mathrm{C}$ in hydrogen in $0.5 \mathrm{~T}$. However, the bump showed up again at about $100 \mathrm{~Hz}$ after the MTJ is annealed at $265^{\circ} \mathrm{C}$ in hydrogen in $7 \mathrm{~T}$. This result indicates that the magnetic fluctuators changed their frequency based on annealing field and temperature. The change in frequencies of the magnetic fluctuators in the MTJ sample may relate to the change of the strength of magnetic pinning sites and is not predictable.

In summary, we studied the low frequency noise of a few different sizes of MTJs in 64 element symmetric bridges. Our results indicate that the magnetic fluctuators in these MTJs change their frequency based on annealing field and temperature. The noise of the MTJs at low frequency could be reduced by annealing in high magnetic field $(7 \mathrm{~T})$ and further improved by annealing in a hydrogen environment.

\section{ACKNOWLEDGMENTS}

This research was supported by the SERDP Grant No. MM-1569, NSF MRSEC Award No. DMR-0213808, and ARO DAAD Grant No. 19-03-1-0298.

${ }^{1}$ J. Scola, H. Polovy, C. Fermon, M. Pannetier-Lecoeur, G. Feng, K. Fahy, and J. M. D. Coey, Appl. Phys. Lett. 90, 252501 (2007).

${ }^{2}$ J. M. Almeida, R. Ferreira, P. P. Freitas, J. Langer, B. Ocker, and W. Maass, J. Appl. Phys. 99, 08B314 (2006).

${ }^{3}$ N. A. Stutzke, S. E. Russek, D. P. Pappas, and M. Tondra, J. Appl. Phys. 97, 10Q107 (2005).

${ }^{4}$ L. Jiang, E. R. Nowak, P. E. Scott, J. Johnson, J. M. Slaughter, J. J. Sun, and R. W. Dave, Phys. Rev. B 69, 054407 (2004).

${ }^{5}$ K. B. Klaassen, J. C. L. van Peppen, and X. Xing, J. Appl. Phys. 93, 8573 (2003).

${ }^{6}$ W. Kyu Park, J. S. Moodera, J. Taylor, M. Tondra, J. M. Daughton, A. Thomas, and H. Brückl, J. Appl. Phys. 93, 7020 (2003).

${ }^{7}$ N. Smith and P. Arnett, Appl. Phys. Lett. 78, 1448 (2001).

${ }^{8}$ S. Ingvarsson, G. Xiao, S. S. P. Parkin, W. J. Gallagher, G. Grinstein, and R. H. Koch, Phys. Rev. Lett. 85, 3289 (2000).

${ }^{9}$ S. Ingvarsson, G. Xiao, R. A. Wanner, P. Trouilloud, Yu Lu, W. J. Gallagher, A. Marley, K. P. Roche, and S. S. P. Parkin, J. Appl. Phys. 85, 5270 (1999).

${ }^{10}$ E. R. Nowak, R. D. Merithew, M. B. Weissman, I. Bloom, and S. S. P. Parkin, J. Appl. Phys. 84, 6195 (1998).

${ }^{11}$ M. B. Weissmam, Rev. Mod. Phys. 60, 537 (1988).

${ }^{12}$ P. Dutta and P. M. Horn, Rev. Mod. Phys. 53, 497 (1981). 